\title{
CVM analysis of crossover in the semi-infinite Ising model
}

\author{
Alessandro Pelizzola \\ Dipartimento di Fisica and Unitá INFM, Politecnico di Torino, I-10129 Torino, Italy
}

\begin{abstract}
The crossover behavior of the semi-infinite three dimensional Ising model is investigated by means of Padé approximant analysis of cluster variation method results. We give estimates for ordinary critical as well as for multicritical exponents, which are in very good agreement with extensive Monte Carlo simulations.
\end{abstract}

Keywords:

Critical phenomena: surface

Ising model: semi-infinite

Crossover

Multicritical point

Address:

Alessandro Pelizzola

Dipartimento di Fisica

Politecnico di Torino

Corso Duca degli Abruzzi 24

I-10129 Torino, ITALY

Tel. $+39-11-5647318$

Fax. +39-11-5647399

E-mail: pescarmona@polito.it 
The present paper reports on a preliminary investigation of the multicritical behavior of the semi-infinite three dimensional Ising model by means of a recently proposed technique [1] which combines the off-critical great accuracy of the cluster variation method (CVM) [2 [4 with the analysis tools for critical behavior based on the well-known Padé approximants [5].

The semi-infinite Ising model (see Ref. [6] for a review) is described by the (reduced) hamiltonian

$$
\beta H=-J_{\mathrm{s}} \sum_{\langle i j\rangle \subset \partial \Lambda} s_{i} s_{j}-J \sum_{\langle k l\rangle \not \partial \Lambda \Lambda} s_{k} s_{l},
$$

where $s_{i}= \pm 1$ is an Ising spin at the site $i$ of the lattice $\Lambda, \partial \Lambda$ is the free surface of the lattice, $J_{\mathrm{s}}, J>0$ are the surface and bulk nearest neighbor interaction strengths, respectively and $\beta=1 / k_{B} T$, with $k_{B}$ Boltzmann's constant and $T$ absolute temperature. In the following, we will take $\Lambda$ to be a simple cubic lattice and $\partial \Lambda$ a (100) free surface.

The phase diagram of this model is well-known. For $\Delta \equiv J_{\mathrm{s}} / J-1<\Delta_{c}$ we have the so-called ordinary transition at the bulk critical temperature, with simultaneous (but with different critical exponents) disordering of bulk and surface. For $\Delta>\Delta_{c}$ we have first the extraordinary transition at the bulk critical temperature, at which only the bulk disorders, and then the surface transition, at a higher temperature, at which the surface disorders. For $\Delta=\Delta_{c}$ we have a multicritical point at the bulk critical temperature, at which the special transition takes place. The surface transition is in the two dimensional Ising universality class and, in the vicinity of the special point, its critical temperature has the power-law behavior

$$
T_{\mathrm{cs}}-T_{\mathrm{cb}} \sim\left(\Delta-\Delta_{c}\right)^{\phi},
$$

where $\phi$ is the crossover exponent associated to the multicritical point.

Our purpose is to show that reliable estimates for the location of the multicritical point and the crossover exponent, as well as other critical exponents, can be obtained by means of a new technique [] which relies on a Padé approximant analysis of CVM results. The basic idea is to find a region, far enough from the critical point, where the quantity of interest can 
be obtained with a given accuracy (say, $10^{-5}$ ) from the CVM, and then to construct $[L, M]$ Padé approximants for functions like that in Eq. 3 on a suitably chosen set of $L+M+1$ points in the region of accuracy (see Ref. [1] for more details).

In what follows, we shall use the $4 \times N$ approximation which we have introduced in Ref. [7]. Here the semi-infinite lattice is approximated by a film of $N$ layers, with the topmost layer representing the free surface, and the bottom layer constrained to the bulk, which in turn is studied in the cube approximation. In this system the maximal clusters for the CVM are chosen as those clusters with $4 N$ sites, formed by a column of $N-1$ elementary cubes. The approximation is formally similar to the square (Kramers-Wannier) approximation for the square lattice and in fact reduces to it for vanishing bulk interaction.

By means of the $4 \times N$ CVM approximation with $N=4$ (but we had to use also $N=5$ for $\Delta<0$ and low $J$ ), we determined the behavior of the surface layer magnetization $m_{1}$ as a function of the temperature for $J>J_{\min }=0.30$ and several values of $\Delta$, in the range $-0.50 \leq \Delta \leq 0.60$. In this region the maximum error on $m_{1}$ is estimated [1],7] to be about $10^{-5}$ or less.

We then determined, following the procedure proposed in Ref. [1], Padé approximants for the function

$$
\beta_{1}^{*}(z, \Delta)=\left(z_{\mathrm{c}}-z\right) \frac{d}{d z} \ln m_{1}(z, \Delta)
$$

where $z=e^{-J}$ and $z_{\mathrm{c}} \simeq 0.801$ [1,, ] . We obtained very stable Padé tables for $\beta_{1}^{\text {eff }}(\Delta)=$ $\beta_{1}^{*}\left(z_{\mathrm{c}}, \Delta\right)$, and our estimates are plotted in Fig. 1, which shows very good agreement with available Monte Carlo data [9]. As already mentioned in Ref. [1] the exponent for the ordinary transition is estimated as $\beta_{1} \simeq 0.78$, again in very good agreement with Monte Carlo estimates [9, 10].

For $\Delta>0$, the effective exponent is appreciably different from its limiting value $\beta_{1}$, indicating that the crossover region is quite large. This allows us to study the multicritical behavior of our model by analyzing the behavior of the critical amplitude $B_{1}(\Delta)$ defined by

$$
m_{1}(z, \Delta) \sim B_{1}(\Delta)\left(z_{\mathrm{c}}-z\right)^{\beta_{1}}, \quad z \rightarrow z_{\mathrm{c}}^{-}
$$


In fact, since near the multicritical point one has

$$
m_{1}(z, \Delta) \simeq\left(z_{\mathrm{c}}-z\right)^{\beta_{1}^{\mathrm{m}}} \tilde{m}_{1}(x), \quad x=\frac{\Delta_{\mathrm{c}}-\Delta}{\left(z_{\mathrm{c}}-z\right)^{\phi}},
$$

the critical amplitude $B_{1}(\Delta)$ must diverge as $\left(\Delta_{c}-\Delta\right)^{-\left(\beta_{1}-\beta_{1}^{\mathrm{m}}\right) / \phi}$. By fitting this behavior we found $\Delta_{c} \simeq 0.515$ and $\left(\beta_{1}-\beta_{1}^{\mathrm{m}}\right) / \phi \simeq 1.39$, which, using $\beta_{1}^{\mathrm{m}}=\beta_{1}^{\text {eff }}\left(\Delta_{\mathrm{c}}\right) \simeq 0.19$, yields $\phi \simeq 0.42$. The calculated amplitudes, together with the theoretical curve, are shown in Fig. 2, where it can be seen that the fit is remarkably accurate.

By comparing these results with those from extensive Monte Carlo simulations in Tab. 1 we see that our values for $\Delta_{\mathrm{c}}$ and $\beta_{1}^{\mathrm{m}}$ are again in very good agreement with previous results, while for the crossover exponent $\phi$, where the situation is less clear, we obtained a value which turns out to be closer to Ruge et al. result than to Binder and Landau one. As a general remark, our technique seems to be fairly more accurate than the second order $\epsilon$-expansion. It is also quite simple and does not require very large CPU times $(\sim 25$ hours on a DEC Alpha for this work). 


\section{REFERENCES}

[1] A. Pelizzola, Phys. Rev. E 49 (1994) R2503.

[2] R. Kikuchi, Phys. Rev. 81 (1951) 988.

[3] G. An, J. Stat. Phys. 52 (1988) 727.

[4] T. Morita, J. Stat. Phys. 59 (1990) 819.

[5] A.J. Guttmann, in Phase transition and Critical Phenomena, vol. 13, edited by C. Domb and J.L. Lebowitz (Academic, London, 1989) and refs. therein.

[6] K. Binder, in Phase transition and Critical Phenomena, vol. 8, edited by C. Domb and J.L. Lebowitz (Academic, London, 1983).

[7] A. Pelizzola, submitted to Physica A.

[8] A.M. Ferrenberg and D.P. Landau, Phys. Rev. B 44 (1991) 5081.

[9] D.P. Landau and K. Binder, Phys. Rev. B 41 (1990) 4633.

[10] C. Ruge et al., J. Stat. Phys. 73 (1993) 293.

[11] H.W. Diehl and S. Dietrich, Z. Phys. B 42 (1981) 65. 


\section{TABLES}

TABLE I. Comparison with Monte Carlo simulations and $\epsilon$-expansion.

\begin{tabular}{lccc}
\hline \hline & $\Delta_{\mathrm{c}}$ & $\beta_{1}^{\mathrm{m}}$ & $\phi$ \\
\hline Present work & 0.515 & 0.19 & 0.42 \\
Landau and Binder [9] & 0.52 & 0.18 & 0.461 \\
Ruge et al. [10] & 0.5004 & 0.237 & 0.59 \\
$\epsilon$-expansion [11] & - & 0.245 & 0.68 \\
\hline \hline
\end{tabular}




\section{FIGURES}

FIG. 1. Effective exponent for the surface layer magnetization. Present work $(\bullet$, solid line is a guide to the eye) and Monte Carlo (×, from Ref. [9]).

FIG. 2. Critical amplitude of the surface layer magnetization. The solid line is our fit. 
Fig. 1

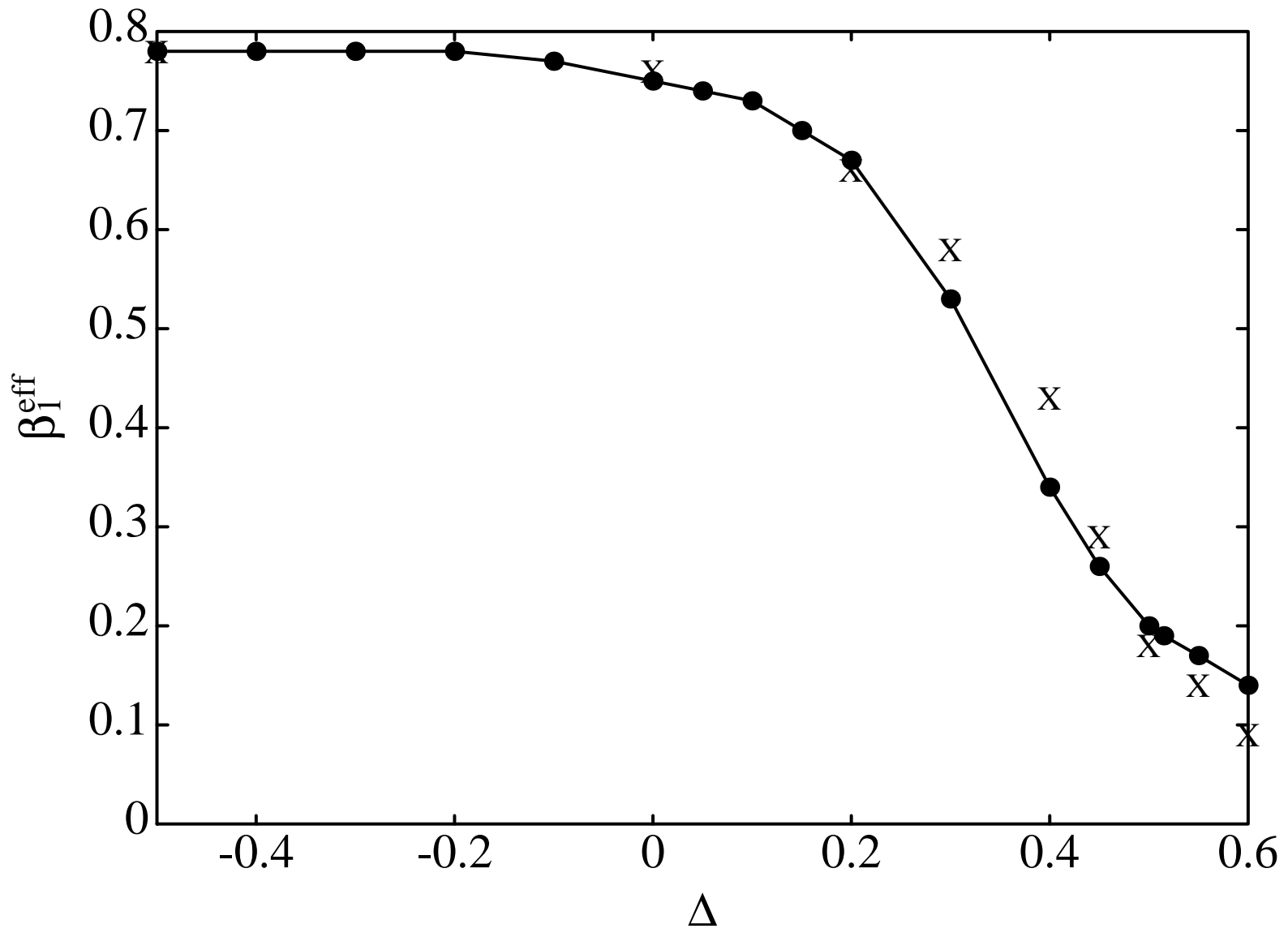


Fig. 2

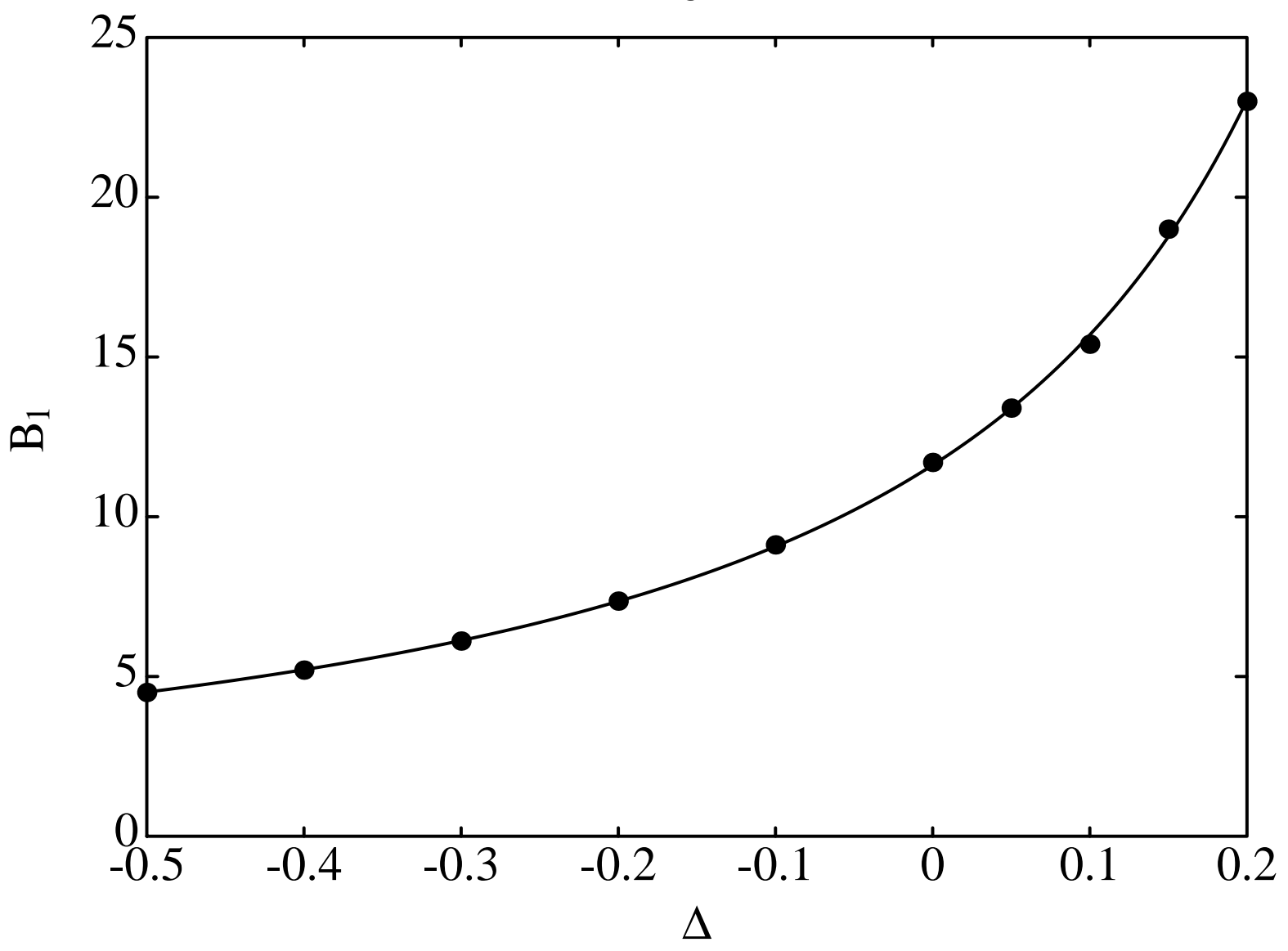

